\title{
Innovations
}

\section{Impact of fluorescent color temperature on attention deficit hyperactivity disorder (ADHD) subjects: A FMRI study}

\author{
November 17-18, 2018 \\ Doha, Qatar
}

Medical Humanities in the Middle East Conference

\section{Authors}

Cherif M. Amor ${ }^{* *}$; Michael O’Boyle ${ }^{2}$; Debajyoti Pati ${ }^{2}$

\section{Introduction}

Emerging neuroscience research shows that environmental-related activity such as perception, wayfinding, cognition, and their behavioral consequences-anxiety, stress, happiness, and arousal-are reflected both in the structures and electro-chemical processes of the brain (Amor, Pati, Oboyle, Duy, \& Hou, 2014; Eberhard, 2007; Mallgrave, 2011; Pati, O’Boyle, Amor, Hou, Valipoor, \& Fang, 2014; Swanson, 2011; Zeisel, 2006).

For ADHD subjects, functional neuroimaging suggests increased neural activity in sensory areas of the brain normally associated with stimulus driven processing, and decreased activity in areas normally associated with higher cognitive processing. Hence, people with ADHD show unusually high activation in ventral occipital areas and abnormally low activation in prefrontal and parietal areas (Baron-Cohen, S. 2004). These findings remain controversial and debatable (Dawson, G. \& Watling, R. 2000; O'Neil, Meena \& Robert Jones, 2007) particularly in that the impact of environmental stimuli (light, color, and sound) were not included. Furthermore, there is a growing body of debatable environment behavior literature relative to the impact of fluorescent lighting on cognitive, behavioral, and psychosocial outcomes, and little is known about the correlation between neural activity and the impact of fluorescent lighting color temperature on indoor behavioral
'Virginia Commonwealth University-Qatar, Doha, Qatar

${ }^{2}$ Texas Tech University, Texas, USA

*Email: mcamor@vcu.edu

Cherif Mohamed Amor iD https://orcid.org/0000-0002-23062090

\section{Cite this article as:}

Amor CM, O'Boyle M, Pati D. Impact of fluorescent color temperature on attention deficit hyperactivity disorder (ADHD) subjects: A FMRI study. In: Weber AS, Verjee MA, editors. Proceedings of the lst International Conference on Medical Humanities in the Middle East [Internet]; 2018 Nov 17-18: Doha, Qatar: Innovations in Global Health Professions Education; 2019 March. p. 24-26. (Innovations in Global Health Professions Education; vol. 2019, spec. no.: 1). https://dx.doi.org/10.20421/ ighpe2019.01.08

This is an open access article distributed under the terms of the Creative Commons Attribution license CC BY 4.0, which permits unrestricted use, distribution and reproduction in any medium, provided the original work is properly cited. 
outcomes. This study is raising the question of whether there are fluorescent lighting Correlated Color Temperatures (CCT) that have more impact on the activation of the cognitive areas of the brain of ADHD subjects. Specifically, are there cognitive neural activations associated with exposure to specific lighting colors?

\section{Purpose of the Study}

The purpose of this research is to: 1) explore and compare behavioral and neural responses of ADHD subjects and a control group, when exposed to 3 types of fluorescent lighting color temperatures: a) Warm White WW with a 2800 CCT, b) Cool White CW with a 4100 CCT, and c) Daylight DX with 6000 CCT), when presented under three different settings-commercial, educational, and healthcare, and 2) explore the impact of different color temperatures on the activation of the prefrontal and parietal areas, brain regions associated with cognition that experience minimal neural activity for people with ADHD. This research aims at providing behavioral and neural benchmark data relative to lighting color temperature that facilitate or inhibit cognitive skills of ADHD subjects.

\section{Methodology/Procedure}

A purposive sampling was used to generate twenty subjects; taking into account gender, age, ethnicity, brain lateralization, as well as the exclusion of color blindness. The participants underwent: 1) an anatomical scan and 2) a functional scan, using Functional Magnetic Resonance Imaging (fMRI) technology, while a random sequence of three types of illustrations from the aforementioned categories were projected by a computer controlled visual presentation system. Each image category included 6 images for a total of 18 images that every participant evaluates. Concomitantly, the participants were asked to respond to each image by fiber optic button device, rating each image on a seven-point Likert satisfaction scale of $1=$ very dissatisfied and $7=$ very satisfied. Behavioral data was analyzed using t-test factor analysis and one-way analysis of variance, while the neural data maps were analyzed using FSL Neuroimaging Software.

\section{Findings/Outcomes}

Findings suggest that contrary to precedents (seeing color activates the ventral occipital and fusiform), the Warm White color temperature (2800K) did not show activation of the occipital cortex. This may indicate disinterest or dissatisfaction with the warm spectrum. Important to note that the following structures were activated under the Cool White spectrum (4100K): the Superior Temporal Gyrus implicated in critical structure of social interaction; the Middle Frontal Gyrus implicated in semantic and analytical tasks; and the Angular Gyrus implicated in memory retrieval-all areas associated with brain cognitive functions. Furthermore, under the daylight color spectrum, the cerebellum-emerging neuroscience indicates that it is involved in cognitive brain processes-has been activated. These neural findings, in support of behavioral findings, suggest a higher satisfaction with cool white and daylight full spectrum than with the warm spectrum.

\section{Conflicts of interest: None.}

Funding sources: Cherif Amor, Michael O'Boyle, and Debajyoti Pati were funded by Texas Tech University, College of Human Sciences

\section{References}

1. Doidge $\mathrm{N}$. The brain that changes itself: Stories of personal triumph from the frontiers of brain science. New York, NY: Viking; 2007. $427 \mathrm{p}$.

2. Eberhard JP. Architecture and the brain: A new knowledge base from neuroscience. Atlanta, GA: Greenway Communications; 2007. $135 \mathrm{p}$.

3. Mallgrave HF. The architect's brain: Neuroscience, creativity, and architecture. Chichester, England: Wiley-Blackwell; 2011.

4. Marcus CC, Barnes M. Healing gardens: Therapeutic benefits and design recommendations. New York, NY: Wiley; 1999. $610 \mathrm{p}$.

5. Rashid M, Zimring C. A review of the empirical 
literature on the relationships between indoor environment and stress in health care and office settings: problems and prospects of sharing evidence. Environ Behav. 2008 Mar 1;40(2):151-190. doi:10.1177/0013916507311550.

6. Swanson LW. Brain architecture:

Understanding the basic plan. 2nd ed. Oxford, England: Oxford University Press; 2012. 331 p.

7. Zeisel J. Inquiry by design: Environment/ behavior/neuroscience in architecture, interiors, landscape, and planning. Rev. ed. New York, NY: W. W. Norton \& Company; 2006. $400 \mathrm{p}$.

\section{About the Author}

Dr. Cherif Mohamed Amor, Ph.D., EDRA, IDEC, \& IIDA, joined Virginia Commonwealth University-Qatar in 2013 to serve as the chair of the Department of Interior Design. He formerly served consecutively as the director of the Interior and Environmental Design graduate program and chair of the Department of Design, Texas Tech University. He earned a Ph.D. in Environmental Design with a specialization in the "semantics of the built environment", from the University of Missouri Columbia (2000). Cherif serves as a reviewer of several design journals/publications. He also serves as a site visitor for the Council for Interior Design Accreditation (CIDA), and as the chair of the interior design network within the Environmental Design Research Association (EDRA). Furthermore, Cherif serves as a curriculum review consultant for the Texas International Education Consortium (TIEC) and as curricula design consultant for Middle East Design Colleges. During the last five years, Cherif has been the recipient and nominee of fifteen teaching, research, and service awards/recognitions. 